\title{
CENTERS OF CURVATURE
}

\author{
BY GERALD FREILICH
}

Communicated by L. Zippin, November 18, 1965

Professor Marston Morse has asked the question as to what can be said concerning the set of centers of curvature of a plane $C^{\prime \prime}$ curve; specifically how dense can this set be? The corresponding question for a $C^{\prime \prime \prime}$ curve is easily disposed of, since roughly two derivatives are used for the curvature leaving one derivative to work with. The difficulty in the $C^{\prime \prime}$ case may then be compared with the far greater pathology that a continuous curve can exhibit in contrast to a $C^{\prime}$ curve. However, as a corollary to our Theorem below, which is presented in the Euclidean plane where the essence of the proof can be observed, it follows that the centers of curvature are nowhere dense in the $C^{\prime \prime}$ case, provided the parameter domain is compact. The key ideas are two in number; firstly the use of Jordan content, and secondly the intimate use of the geometry of the situation, i.e. the concept of curvature and circle of curvature.

THEOREM. The centers of curvature of a regular $C^{\prime \prime}$ curve (with compact parameter domain) that lie in a bounded portion of the plane, form a set of zero two-dimensional Jordan content.

Outline of Proof. Without loss of generality, we may take the arc length $s$ as the new parameter and assume the length of the curve is unity. If for convenience we take the initial point at the origin, we may represent the curve parametrically as:

$$
P(s)=f(s)+i \cdot g(s), \quad 0 \leqq s \leqq 1,
$$

with $P(0)=0, f \in C^{\prime \prime}, g \in C^{\prime \prime},\left(f^{\prime}(s)\right)^{2}+\left(g^{\prime}(s)\right)^{2}=1$ for $0 \leqq s \leqq 1$.

$$
\begin{aligned}
& \text { Let } \\
& S(0, r)=\{x+i y:|x+i y|<r\}
\end{aligned}
$$

$\alpha(s)=\arg P^{\prime}(s)$, chosen as a continuous function of $s$,

$K(s)=\alpha^{\prime}(s)=$ curvature at $s$,

$R(s)=|K(s)|^{-1}$, with $0^{-1}$ undefined,

$C(s)=$ center of curvature corresponding to $P(s)$.

Since $K(s)$ is continuous on $0 \leqq s \leqq 1$, choose $N>1$ so that $|K(s)|<N$ for $0 \leqq s \leqq 1$. Fix $M$ and $\epsilon$ as arbitrary numbers subject to the conditions $M>2$ and $(2 N)^{-1}>\epsilon>0$. Define, for $0 \leqq s \leqq 1$,

$$
R_{M}(s)=\min (R(s), 3 M) \text { with } R_{M}(s)=3 M \text { if } K(s)=0 .
$$

Choose $\delta$ so that $0<\delta<1$ and 
$\left(0 \leqq s_{1} \leqq 1,0 \leqq s_{2} \leqq 1,\left|s_{1}-s_{2}\right|<\delta\right) \Rightarrow\left(\left|R_{M}\left(s_{1}\right)-R_{M}\left(s_{2}\right)\right|<\epsilon\right)$.

Let $0 \leqq s \leqq 1$. If $C(s) \notin S(0,(1+\epsilon) M)$, choose $\delta_{s}>0$ so that

$$
\left(0 \leqq s^{\prime} \leqq 1,\left|s^{\prime}-s\right|<\delta_{s}\right) \Rightarrow\left(C\left(s^{\prime}\right) \notin S(0, M)\right) .
$$

If $C(s) \in S(0,(1+\epsilon) M)$, choose $\delta_{s}>0$ so that:

(i) $\delta_{s}<\delta$,

(ii) $\left(0 \leqq s^{\prime} \leqq 1,\left|s^{\prime}-s\right|<\delta_{s}\right) \Rightarrow\left(\left|\alpha\left(s^{\prime}\right)-\alpha(s)-\alpha^{\prime}(s)\left(s^{\prime}-s\right)\right|<\epsilon\left|s^{\prime}-s\right|\right)$,

(iii) $\left(0 \leqq s^{\prime} \leqq 1,\left|s^{\prime}-s\right|<\delta_{s}\right) \Rightarrow\left(\left|P_{s}\left(s^{\prime}\right)-P\left(s^{\prime}\right)\right|<\epsilon\left|s^{\prime}-s\right|\right)$,

where

$$
P_{s}\left(s^{\prime}\right)=P(s)+\left(\alpha^{\prime}(s)\right)^{-1} \cdot\left(e^{i(\alpha(s)+\pi / 2)}-e^{i\left(\alpha(s)+\pi / 2+\alpha^{\prime}(s)\left(s^{\prime}-s\right)\right.}\right) .
$$

By the Heine-Borel theorem, choose a finite, minimal covering of $[0,1]$ by intervals of the form $\left(s-\delta_{s}, s+\delta_{s}\right)$; minimal in the sense that no refinement is a covering. Henceforth, $s$ will refer to the center of one of the intervals of the minimal covering. Then $\sum \delta_{s} \leqq 3$. We next define, for those (and only those) $s$ such that $C(s) \in S(0,(1+\epsilon) M)$, a rectangle $Q_{s}$ as follows:

With $(a+i b) \bullet(c+i d)$ defined as $a c+b d$, then $x+i y \in Q_{\mathrm{s}} \Leftrightarrow\left\{\begin{array}{l}\left|(x+i y-C(s)) \bullet e^{i \alpha(s)}\right|<\epsilon \delta_{s}(1+2 M+N+\epsilon) \text {, and } \\ \left|(x+i y-C(s)) \bullet e^{i(\alpha(s)+\pi / 2)}\right|<\epsilon\left((2 M+1) \delta_{s}+1\right) .\end{array}\right.$

Now if $0 \leqq s^{\prime} \leqq 1$ and $\left|s^{\prime}-s\right|<\delta_{s}$, then $C\left(s^{\prime}\right) \in Q_{s}$. The idea used to prove that $C\left(s^{\prime}\right) \in Q_{s}$ proceeds in the following stages: First approximate $P\left(s^{\prime}\right)$ by $P_{s}\left(s^{\prime}\right)$. From $P_{s}\left(s^{\prime}\right)$ proceed a distance $\left(\alpha^{\prime}\left(s^{\prime}\right)\right)^{-1}$ in the direction $\alpha\left(s^{\prime}\right)+\pi / 2$, arriving at a point which we label $D\left(s^{\prime}\right)$. Label by $E\left(s^{\prime}\right)$ the point obtained by proceeding from $P_{s}\left(s^{\prime}\right)$ a distance $\left(\alpha^{\prime}(s)\right)^{-1}$ in the direction $\alpha\left(s^{\prime}\right)+\pi / 2$. Note that if one proceeded a distance $\left(\alpha^{\prime}(s)\right)^{-1}$ in the direction $\alpha(s)+\alpha^{\prime}(s)\left(s^{\prime}-s\right)+\pi / 2$, one would arrive at $C(s)$. Hence $\left|C(s)-E\left(s^{\prime}\right)\right| \leqq\left|\alpha^{\prime}(s)\right|^{-1} \epsilon \delta_{s}$. Since

$$
R(s) \leqq|P(s)|+(1+\epsilon) M \leqq 1+(1+\epsilon) M \leqq\left(\frac{3}{2}+\epsilon\right) M<2 M,
$$

it follows that

$$
\left|C(s)-E\left(s^{\prime}\right)\right|<2 M \epsilon \delta_{s} .
$$

Since $\left|R\left(s^{\prime}\right)-R(s)\right|<\epsilon$ and

$$
\left|\alpha\left(s^{\prime}\right)-\alpha(s)\right|<\left(\left|\alpha^{\prime}(s)\right|+\epsilon\right)\left|s^{\prime}-s\right| \leqq(N+\epsilon) \delta_{s},
$$

it follows that

$$
\left|\left(E\left(s^{\prime}\right)-D\left(s^{\prime}\right)\right) \bullet e^{i \alpha(s)}\right| \leqq \epsilon \sin (N+\epsilon) \delta_{s}<\epsilon(N+\epsilon) \delta_{s} .
$$

Since $\left|C\left(s^{\prime}\right)-D\left(s^{\prime}\right)\right|=\left|P\left(s^{\prime}\right)-P_{s}\left(s^{\prime}\right)\right|<\epsilon \delta_{s}$, it follows that 


$$
\begin{aligned}
& \left|\left(C\left(s^{\prime}\right)-C(s)\right) \bullet e^{i \alpha(s)}\right| \\
& \quad \leqq\left|C\left(s^{\prime}\right)-D\left(s^{\prime}\right)\right|+\left|\left(D\left(s^{\prime}\right)-E\left(s^{\prime}\right)\right) \bullet e^{i \alpha(s)}\right|+\left|E\left(s^{\prime}\right)-C(s)\right| \\
& \quad<\epsilon \delta_{s}(1+2 M+N+\epsilon) .
\end{aligned}
$$

Also

$$
\begin{aligned}
\mid\left(C\left(s^{\prime}\right)-\right. & C(s)) \bullet e^{i(\alpha(s)+\pi / 2)} \mid \\
& \leqq\left|C\left(s^{\prime}\right)-D\left(s^{\prime}\right)\right|+\left|D\left(s^{\prime}\right)-E\left(s^{\prime}\right)\right|+\left|E\left(s^{\prime}\right)-C(s)\right| \\
& \leqq \epsilon\left((2 M+1) \delta_{s}+1\right) .
\end{aligned}
$$

Now the area of $Q_{s}$ is $4 \epsilon^{2} \delta_{s}(1+2 M+N+\epsilon)\left((2 M+1) \delta_{s}+1\right)$ $\leqq 8 \epsilon^{2} \delta_{s}(2+2 M+N)(M+1)$. Since $\sum \delta_{s} \leqq 3$, then

$$
\sum \text { area } Q_{s} \leqq 24 \epsilon^{2}(2+2 M+N)(M+1),
$$

which approaches zero as $\epsilon$ approaches zero. Since the rectangles $Q_{8}$ form a finite covering of

$$
\{C(s): 0 \leqq s \leqq 1\} \cap S(0, M),
$$

the latter set must have zero Jordan content.

COROLlaRY. The centers of curvature of a $C^{\prime \prime}$ regular curve (with compact parameter domain) are nowhere dense in the plane.

The City College, The City University of New York 